\title{
Parâmetros eritrocitários do jundiá (Rhamdia quelen) submetido à dieta com diferentes níveis de proteína
}

\author{
Erythrocytic parameters of silver catfish (Rhamdia quelen) as \\ affected by different protein level diets
}

\author{
Sabrina Ortiz Camargo ${ }^{1}$ Juvêncio Luís Pouey ${ }^{2}$ Clarice Martins $^{3}$
}

\section{RESUMO}

O jundiá é espécie de interesse econômico para região sul do Rio Grande do Sul, pois adapta-se a diferentes ambientes e dietas artificiais, apresentando fácil manejo $e$ boa aceitação comercial. O bom estado nutricional é fator determinante no desenvolvimento dos peixes. A formação das células sangüíneas é dependente da manipulação da dieta. $O$ objetivo deste trabalho foi avaliar o efeito da dieta com diferentes níveis de proteína sobre os parâmetros eritrocitários do jundiá. Foram utilizados 90 peixes, com

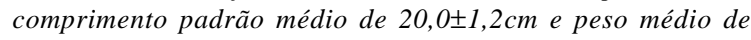
$131,4 \pm 25,9 \mathrm{~g}$. Os animais foram distribuídos em três caixas de $1.000 \mathrm{~L}$, contendo três cestos (80L) cada, com três níveis de proteína bruta (30, 40 e 50\%). Os animais foram amostrados a cada duas semanas, durante 45 dias. Após serem anestesiados, foi realizada a coleta de sangue através de punção caudal, utilizando-se agulha e seringa que contêm EDTA a 10\%. As análises sangüíneas correspondentes à série eritrocitária foram realizadas através de contador automatizado. O nível protéico da dieta influencia os parâmetros eritrocitários, em especial, o número total de eritrócitos, a taxa de hemoglobina e o hematócrito, sendo que o nível de $50 \%$ de PB foi o que mais estimulou a eritropoiese.

Palavras-chave: eritrócitos, nutrição, Pimelodidae, proteína.

\section{ABSTRACT}

Silver catfish is a species of economic interest for the southern Rio Grande do Sul region, Brazil, being well adapted to different environments and artificial diets, showing easy management and good commercial acceptance. Good nutritional status is a determinant factor in fish development. Difference in formation of blood cells is dependent on diet manipulation. The objective of this study was to evaluate the effect of a diet with different protein levels on erythrocytic parameters of silver catfish. Ninety (90) fish were used with an average standard length of $20.01 \pm 1.28 \mathrm{~cm}$ and average weight of $131.4 \pm 25.9 \mathrm{~g}$ distributed in three $1,000 \mathrm{~L}$ tanks, containing three baskets (80L) each, with three levels of crude protein (30, 40 and 50\%). Animals were sampled every two weeks during 45 days. After anesthetization blood was collected through caudal puncture using needle and syringe containing EDTA at $10 \%$. Blood analyses corresponding to the erythrocyte series were performed through automatic counter. Proteic level of diet influences erythrocytic parameters, especially the total number of erythrocytes, hemoglobin rate and hematocrit. The level of 50\% CP showed best stimulation of erythropoiesis.

Key words: erythrocytes, nutrition, Pimelodidae, protein.

\section{INTRODUÇÃO}

O jundiá (Rhamdia quelen) possui distribuição neotropical, encontrando-se desde o sudeste do México até a região sul da Argentina (SILFVERGRIP, 1996). Esta espécie é de grande interesse econômico para região sul do Rio Grande do Sul, pois, adapta-se a diferentes ambientes e dietas artificiais, apresentando fácil manejo e boa aceitação comercial, sendo a espécie nativa local mais promissora para a criação intensiva.

Inúmeros estudos demonstram que diferenças na formação e função das células sangüíneas podem ser indicativas de manipulação da alimentação (GREENE \& SELIVONCHICK, 1990;

${ }^{1}$ E-mail: sabrinaortiz2002@yahoo.com.br

${ }^{2}$ Departamento de Zootecnia, Faculdade de Agronomia Eliseu Maciel, Universidade Federal de Pelotas, Campus Universitário. CP 354, CEP 96001-970, Pelotas, RS, Brasil. Email: juvêncio@ufpel.tche.br

${ }^{3}$ Programa de Pós-graduação em Zootecnia, UFPel, Pelotas, RS, Brasil. E-mail: claritin@ufpel.tche.br 
DUNCAN et al., 1993; WISE et al., 1993). O claro conhecimento da resposta hematológica, para diferentes dietas, pode ser útil para novas formulações. Entretanto, a determinação da exigência nutricional baseia-se no teste de desempenho, em diferentes períodos ambientais (GERHANOVICH \& KISELEV, 1993).

A proteína é o componente mais caro da dieta de peixes. Por esta razão, a exigência protéica é pesquisada e níveis de $35-50 \%$ são os que proporcionam melhor crescimento, dependendo da composição, nível de alimentação, idade e condição fisiológica do peixe. Os aminoácidos são os constituintes fundamentais das proteínas, sendo essenciais para formação e regeneração de grande parte dos tecidos entre eles o sangue (KUBTIZA, 1999).

A falta de padronização de métodos e nomenclatura, as diferentes espécies, o efeito da idade, do sexo, da qualidade de água e os métodos de captura, contribuem para a variabilidade dos parâmetros hematológicos em peixes. Por esta razão, é difícil comparar resultados de diferentes estudos, devendose estes serem determinados para cada condição de cultivo (KLINGER et al., 1996). Este estudo teve o objetivo de avaliar o efeito de diferentes níveis de proteína sobre parâmetros eritrocitários do jundiá, através da avaliação do número total de eritrócitos, hematócrito, taxa de hemoglobina, volume corpuscular médio, hemoglobina corpuscular média e concentração de hemoglobina corpuscular média.

\section{MATERIAL E MÉTODOS}

Foram utilizados 90 jundiás, com comprimento padrão médio de 20,0 \pm 1 ,2cm e peso médio de 131,4 $\pm 25,9 \mathrm{~g}$, provenientes de tanques de cultivo. Os animais foram acondicionados em três caixas, com capacidade para 1.000L de água (termorregulada), contendo três cestos (80L) cada, por três semanas para adaptação ao consumo de ração (comercial - 36\% PB) nestas condições. A qualidade da água foi mantida por aeração constante e renovação diária. Os parâmetros físicos e químicos mantiveram-se nos padrões aceitáveis para a espécie (BALDISSEROTTO, 2003; MELO et al., 2002). A temperatura média foi de $20,1 \pm 1,3^{\circ} \mathrm{C}$, o oxigênio dissolvido $8,0 \pm 1,2 \mathrm{mg} \mathrm{L}^{-1}$ e o $\mathrm{pH}$ $7,2 \pm 0,3$.

Após o período de adaptação, os animais foram alimentados com rações isoenergéticas (3.400Kcal), mas com diferentes níveis de proteína bruta (30, 40 e 50\%), durante 45 dias. As dietas foram calculadas por meio de "software” UFFF (PESTI \& MILLER, 1992) e preparadas tendo como base farelo de soja, de arroz, farinha de peixe e óleo de soja e suplementadas com vitaminas e minerais (4\%). A análise química foi realizada no laboratório de nutrição animal do departamento de Zootecnia - FAEM - UFPel.

A coleta de sangue foi realizada a cada quinze dias, sendo amostrados seis animais, aleatoriamente, por tratamento. Cinco minutos antes da coleta de sangue, os animais foram retirados e anestesiados com benzocaína (3g 20 $\mathrm{mL}^{-1}$ de álcool/ 20L de água) e a coleta foi realizada por punção caudal, com auxílio de seringas (3mL) e agulhas (25x0,7mm) descartáveis contendo EDTA a 10\%. Os principais dados biométricos (peso total, comprimento total e padrão) foram anotados após cada coleta.

As análises da série eritrocitária número total de eritrócitos (NTE - $10^{6} \mu \mathrm{L}^{-1}$ ), hematócrito (HCT - \%), taxa de hemoglobina (HGB - g dL $\mathrm{dL}^{-1}$ ) e índices eritrocitários ou hematimétricos absolutos: volume corpuscular médio (VCM - fL), hemoglobina corpuscular média (HCM - pg) e concentração de hemoglobina corpuscular média (CHCM - $\mathrm{g} \mathrm{dL}^{-1}$ ) foram realizadas por contador automatizado.

O delineamento experimental utilizado foi completamente casualizado, com três tratamentos e três repetições. Os resultados foram avaliados pela análise de variância, usando-se o teste de Tukey, para comparar as médias (5\%) e a relação entre os níveis protéicos e os parâmetros eritrocitários foi descrita pela análise de regressão, pelo programa STATISTICA (1998).

\section{RESULTADOS E DISCUSSÃO}

Na avaliação do desempenho dos animais, observou-se que o ganho de peso não apresentou diferença entre os tratamentos, sendo de 52,5, 48,6 e $40,2 \mathrm{~g}$ para as dietas de 50, 40 e $30 \%$ de $\mathrm{PB}$, respectivamente. A sobrevivência foi de $100 \%$, em todos os tratamentos.

Na avaliação dos parâmetros eritrocitários, observou-se que o número total de eritrócitos (NTE), nos diferentes tratamentos e períodos de observação, oscilou de 1,45 a 3,13 $10^{6} \mu \mathrm{L}^{-1}$, aumentando em todos os níveis de proteína. Aos 45 dias, os valores médios observados foram de 2,65, 2,62 e 2,71 $10^{6} \mu \mathrm{L}^{-1}$ para os níveis de 30, 40 e 50\% de PB, respectivamente. O nível de $50 \%$ foi significativamente superior ao $40 \%$, mas não diferiu da dieta com $30 \% \mathrm{~PB}$, mas a figura 1 demonstra claramente um maior valor para o nível 50\% o que é comprovado nas determinações de HGB e HCT que foram significativamente superiores para este nível (Figuras 2 e 3). Outra possibilidade é a influência do stress no cultivo de jundiá em gaiola (BARCELLOS 


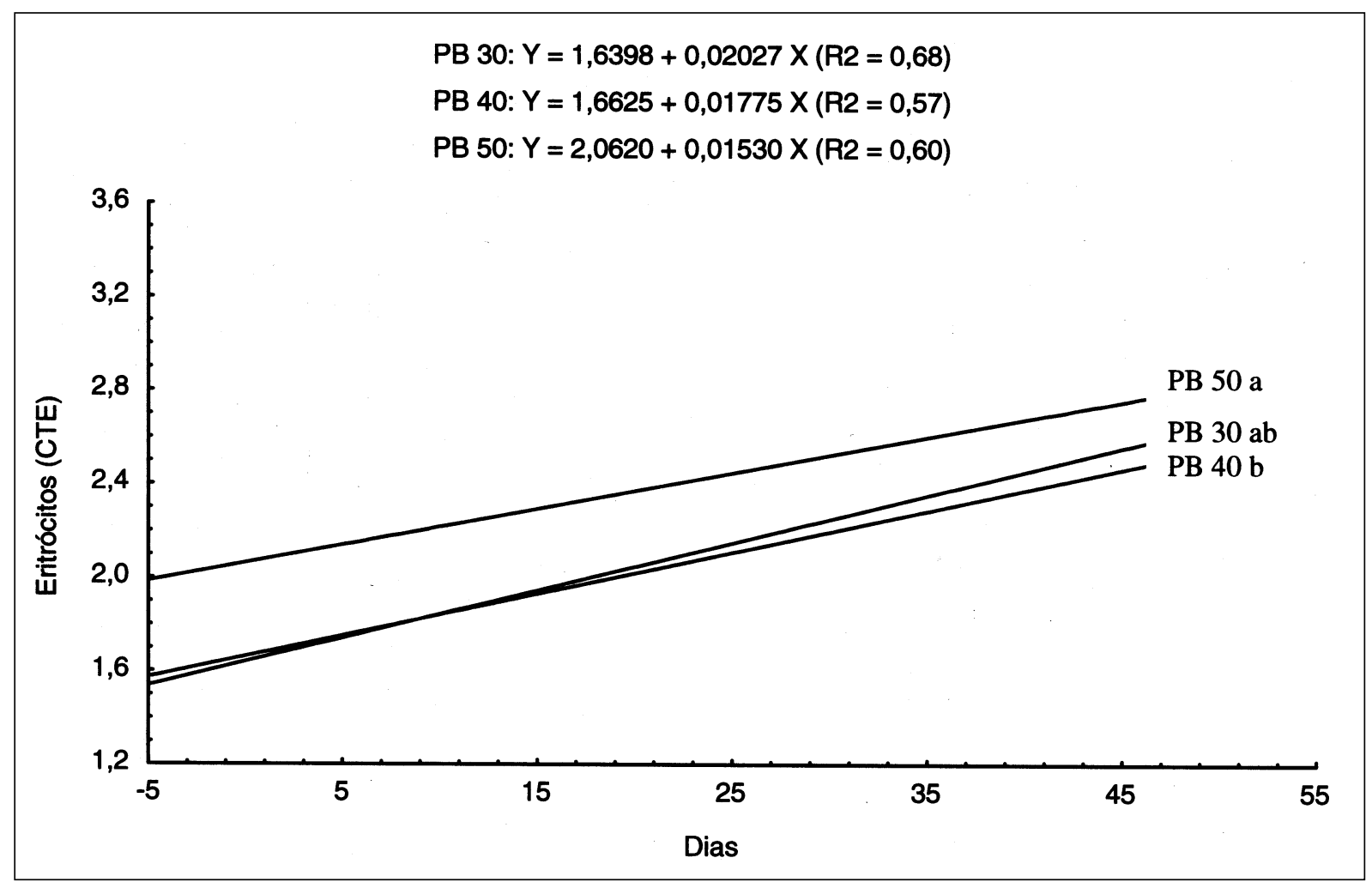

Figura 1 - Equações de regressão, $\mathrm{R}^{2}$ e linhas de tendência das relações entre diferentes níveis de proteína e o número total de eritrócitos (NTE $10^{6} \mathrm{~mL}^{-1}$ ) de jundiá (Rhamdia quelen), durante 45 dias de alimentação em laboratório.

et al., 2004a) e/ou o efeito do stress crônico, visto que os valores deste trabalho foram próximos ao 2,98 $10^{6}$ $\mu \mathrm{L}^{-1}$ encontrado por BARCELLOS et al. (2004b) e superiores ao $1,410^{6} \mu \mathrm{L}^{-1}$ descrito como valor basal (BARCELLOS et al., 2003).

A membrana eritrocitária é constituída por dupla camada lipídica, atravessada por várias proteínas (BAIN, 1997) e quando ocorre deficiência de alguns aminoácidos, a produção de eritrócitos (eritropoiese) pode ser prejudicada, assim como também pode ocorrer decréscimo na produção de hemoglobina, causando anemia (GARCIA-NAVARRO \& PASCHALY, 1994). Portanto o nível de 50\% de PB possibilitou uma boa eritropoiese e foi o que mais estimulou a produção de hemoglobina, assegurando bom estado de saúde aos peixes.

A eritropoiese nos teleósteos é similar a de mamíferos, pois admite-se que os eritrócitos derivam de uma célula fonte e de acordo com o seu grau de maturação essas células são denominadas de eritroblastos basofílicos, eritroblastos policromatofílicos, eritroblastos acidofílicos, reticulócitos e eritrócitos maduros. Entende-se por célula madura aquela que se diferenciou, tendo atingido a possibilidade de desempenhar as suas funções específicas (TAVARES-DIAS \& MORAES, 2004).

A concentração de hemoglobina (HGB) e o percentual de hematócrito (HCT) aumentaram conforme aumentou o nível protéico. A HGB apresentou os valores médios finais (45 dias) de 9,1, 10,1 e 11,0g dL $\mathrm{dL}^{-1}$ para os níveis de 30, 40 e 50\% de PB, respectivamente. Somente a dieta com 50\% de PB foi superior (5\%) aos demais tratamentos (Figura 2).

Os valores médios de hematócrito (HCT), aos 45 dias, foram de 32,0, 34,9 e 36,3\%, para os tratamentos 30, 40 e 50\% PB, respectivamente. Também para o HCT foi observado o maior resultado na dieta mais protéica, sendo somente este diferente (5\%) dos demais (Figura 3). BARCELLOS et al. (2004b) encontraram valores de 40,4 e 33,2\% para animais antes e depois de stress crônico, respectivamente. Observase que pode ter ocorrido influência do local de cultivo, mas o nível protéico foi determinante visto que todos os animais foram cultivados da mesma forma.

Entretanto os índices eritrocitários volume corpuscular médio (VCM), hemoglobina corpuscular média (HCM) e concentração de hemoglobina 


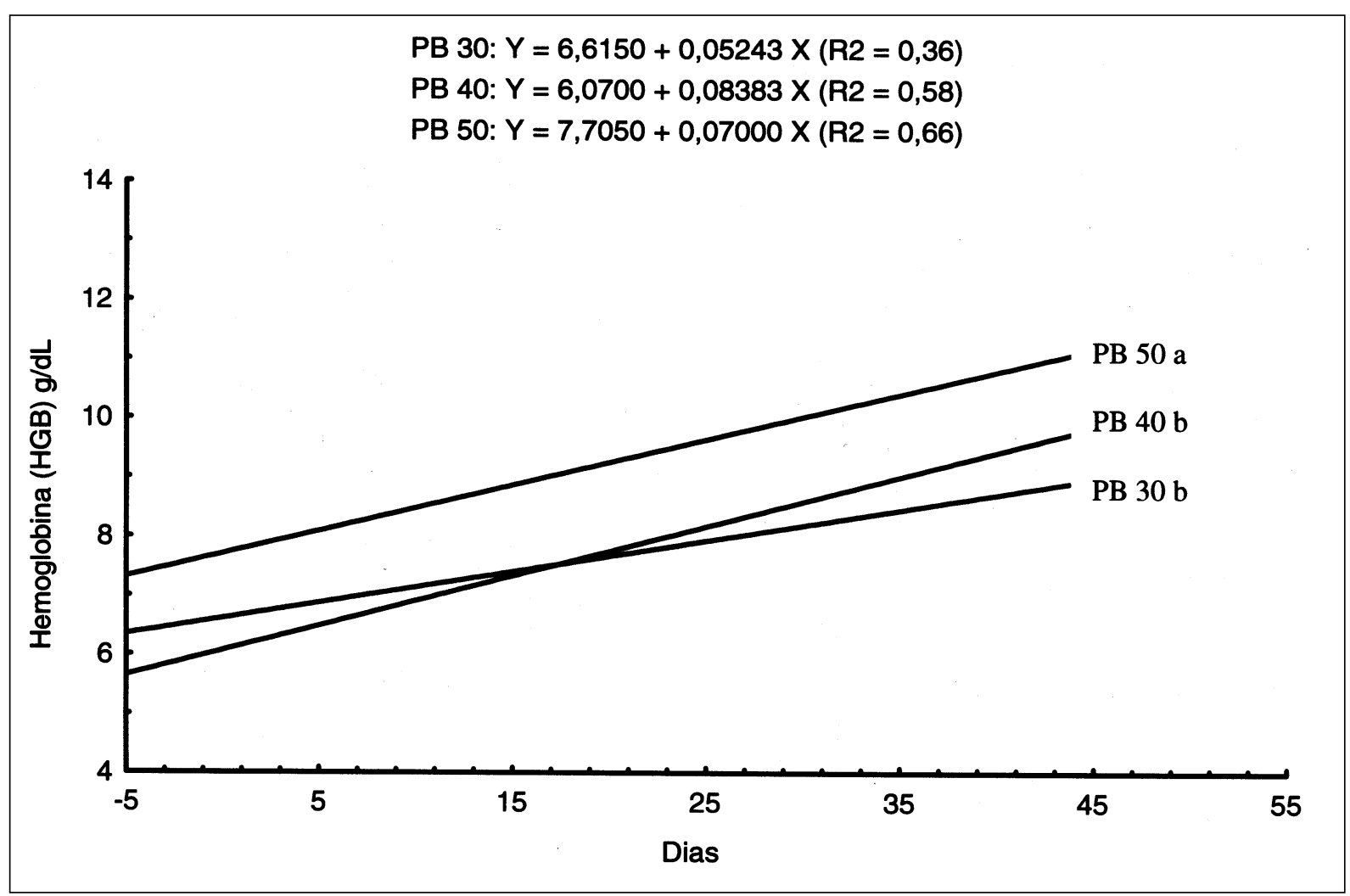

Figura 2 - Equações de regressão, $\mathrm{R}^{2}$ e linhas de tendência das relações entre diferentes níveis de proteína e a concentração de hemoglobina do jundiá (Rhamdia quelen), durante 45 dias de alimentação em laboratório.

corpuscular média (CHCM) não apresentaram diferenças entre os tratamentos. De forma geral observou-se que o aumento da proteína favoreceu o incremento dos parâmetros eritrocitários e o maior nível protéico empregado foi o que apresentou os maiores valores. Tal constatação deve-se provavelmente à composição dos eritrócitos cuja base são as proteínas (GARCIANAVARRO \& PASCHALY, 1994; BAIN, 1997).

Os resultados médios obtidos aos 45 dias para o NTE $\left(2,6510^{6} \mu \mathrm{L}^{-1}\right)$, HGB $(9,1 \mathrm{~g} \mathrm{dL}-1)$ e HCT (32,0\%) na dieta contendo $30 \% \mathrm{~PB}$, foram superiores aos obtidos por TAVARES-DIAS et al. (2002) (NTE 1,95 $10^{6} \mu \mathrm{L}^{-1}$; HGB 6,73g dL $\mathrm{dL}^{-1}$; HCT 26,5\%), na mesma espécie de peixe em cativeiro, com comprimento de $16 \mathrm{~cm}$ e peso médio de $44 \mathrm{~g}$, alimentados com ração comercial contendo 34\% de PB. Esta diferença deve ser devido ao tamanho dos animais.

O HCT para o nível de 30\% de proteína foi inferior aos 35,2\% encontrados por KAVAMOTO et al. (1983), em jundiá proveniente de ambiente natural e superior aos 30,14\% obtidos por FORESTI et al. (1977), em jundiá de cativeiro. Entretanto o HCT encontrado para o nível de 50\% de proteína, aos 45 dias, (36,3\%) foi superior a estes resultados, mas próximo do valor encontrado para os animais de ambiente natural, indicando que eles tinham uma dieta mais protéica, visto ser uma espécie onívora com tendência carnívora.

Os resultados médios obtidos para os índices eritrocitários VCM (133,4fL), HCM (38,3pg) e CHCM (28,7g dL $\left.{ }^{-1}\right)$ estão próximos aos valores encontrados para jundiá por TAVARES-DIAS et al. (2002) (VCM 139,0fL; CHCM 25,9g/dL) e FORESTI et al. (1977) (CHCM 24,92g/dL), mas o VCM encontrado por estes últimos autores foi superior (241,94\%), indicando que seus animais apresentaram um menor número de eritrócitos (NTE 1,7 $10^{6} \mu \mathrm{L}^{-1}$ ), mas de maior volume.

Comparando com outros bagres observase que os resultados de NTE, HGB e HCT são superiores aos encontrados para Ictalurus punctatus (NTE 1,55 $10^{6} \mu \mathrm{L}^{-1}$; HGB 5,9g dL $\mathrm{HL}^{-1}$; HCT 23,9\%) por LIM et al. (2000) e semelhantes aos valores encontrados por LIM \& KLESIUS (2003) (NTE 2,30 $10^{6} \mu \mathrm{L}^{-1}$; HGB 8,4g dL ${ }^{-1}$; HCT 32,4\%). Com relação aos resultados citados para Pimelodus maculatus, observa-se certa semelhança para NTE $\left(2,0410^{6} \mu \mathrm{L}^{-1}\right)$ e HCT (36,3\%), mas o $\operatorname{HGB}\left(7,3 g \mathrm{dL}^{-1}\right)$ foi inferior (RANZANI-PAIVA et al., 2000b). Já para o 


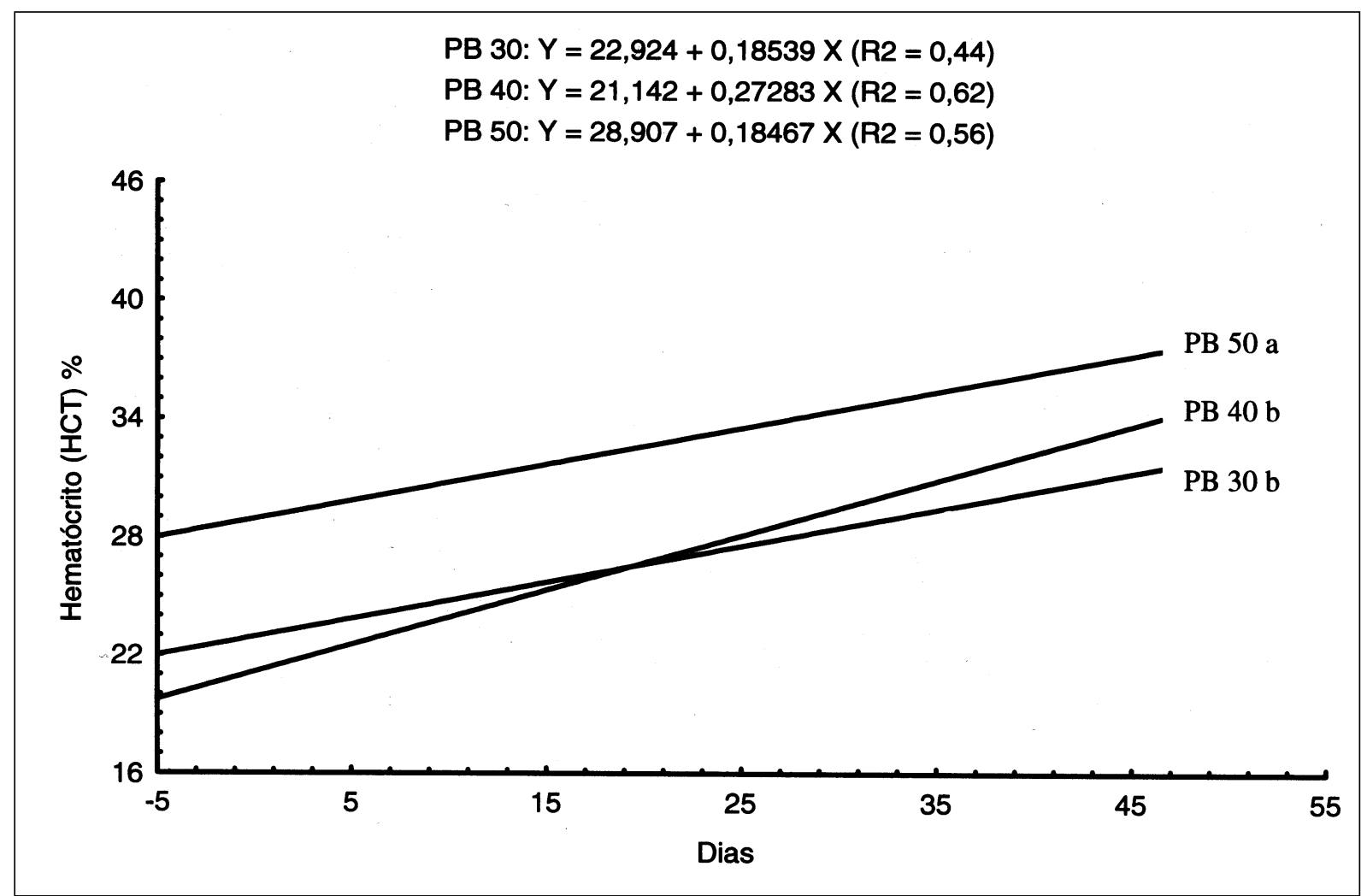

Figura 3 - Equações de regressão, $\mathrm{R}^{2}$ e linhas de tendência das relações entre diferentes níveis de proteína e o percentual de hematócrito de jundiá (Rhamdia quelen), durante 45 dias de alimentação em laboratório.

Pseudoplatystoma fasciatum, os valores médios encontrados foram inferiores (NTE 1,57 $10^{6} \mu \mathrm{L}^{-1}$; HGB 5,65g dL $\mathrm{g}^{-1}$; HCT 27,75\%) (RANZANI-PAIVA et al., 2000a), bem como para o Pseudoplatystoma corruscans (NTE 1,59 $10^{6} \mu \mathrm{L}^{-1}$; HGB 4,9g dL $\mathrm{dL}^{-1}$; HCT 26,0\%) (RANZANI-PAIVA et al., 2000b). Os resultados deste ensaio demonstram que, mesmo havendo variações nos parâmetros eritrocitários entre diferentes espécies e dentro da mesma espécie, o aporte protéico influencia estes parâmetros.

\section{CONCLUSÕES}

O nível protéico da dieta interfere nos parâmetros eritrocitários do jundiá (Rhamdia quelen). O número total de eritrócitos, a concentração de hemoglobina e o percentual de hematócrito do jundiá (Rhamdia quelen) são maximizados com 50\% de proteína bruta.

\section{REFERÊNCIAS}

BAIN, B.J. Células sangüíneas - Um guia prático. 2.ed. Porto Alegre: Artes Médicas, 1997. 334p.
BALDISSEROTTO, B. The culture of silver catfish (Jundiá) (Rhamdia quelen). In: WORLD AQUACULTURE, 2003, Salvador, BA. Anais... Salvador: World Aquaculture Society, 2003. V.1. 480p. p.73.

BARCELLOS, L.J.G. et al. Haematological and biochemical characteristics of male jundiá (Rhamdia quelen Quoy \& Gaimard Pimelodidae): changes after acute stress. Aquaculture Research, v.34, p.1465$1471,2003$.

BARCELLOS, L.J.G. et al. Nursery rearing of jundiá, Rhamdia quelen (Quoy \& Gaimard) in cages: cage type, stocking density and stress response to confinement. Aquaculture, v.232, p.383-394, 2004a.

BARCELLOS, L.J.G. et al. Hematological changes in jundiá (Rhamdia quelen Quoy and Gaimard Pimelodidae) after acute and chronic stress caused by usual aquacultural management, with emphasis on immunosuppressive effects. Aquaculture, v.237, p.229-236, 2004 b.

DUNCAN, P.L. et al. Dietary folate requirement determined for channel catfish, Ictalurus punctatus. Journal of Nutrition, v.123, p.888-1897, 1993.

FORESTI, F. et al. Medidas de alguns parâmetros morfológicos e fisiológicos do sangue de bagre (Rhamdia hilarii, Valenciannes, 1840) (Pisces: Pimelodidae). Ciência e Cultura, Rio de Janeiro, v.29, n.7, p.580, 1977.

Ciência Rural, v.35, n.6, nov-dez, 2005. 
GARCIA-NAVARRO, C.E.K.; PASCHALY, J.R. Hematologia veterinária. São Paulo:Varela, 1994. 161p.

GERSHANOVICH, A.D.; KISELEV, G.A. Growth and hematological response of sturgeon hybrids Russian sturgeon (Acipenser guldenstadti) x beluga (Huso huso L.) to protein and lipid contents in the diet. Comparative Biochemistry Physiology, v.106A, n.3, p.581-586, 1993.

GREENE, D.H.S.; SELIVONCHICK, D.P. Effects of dietary vegetable, animal and marine lipids on muscle lipid and hematology of rainbow trout (Oncorhynchus mykiss) Aquaculture, v.89, p.165-182, 1990.

KAVAMOTO, E.T. et al. Estudos hematológicos em “bagre" Rhamdia hilarii (Valenc. 1840) teleósteos no estádio de desenvolvimento gonadal maduro. Boletim do Instituto de Pesca, São Paulo, v.10, p.53-60, 1983

KLINGER, R.C. et al. Effects of dietary lipid on the hematology of channel catfish (Ictalurus punctatus). Aquaculture, v.47, p.225-233, 1996.

KUBITZA, F. Nutrição e alimentação dos peixes cultivados. 3.ed. Jundiaí : Degaspari, 1999. 123p.

LIM, C. et al. Interaction between dietary levels of iron and vitamin $\mathrm{C}$ on growth, hematology, immune response and resistance of channel catfish (Ictalurus punctatus to Edwardsiella ictaluri challenge. Aquaculture, New York, v.185, p.313-327, 2000.

LIM, C.; KLEISUS, P.H. Influence of dietary levels of magnesium on growth, tissue mineral content, and resistance of channel catfish Ictalurus punctatus challenged with Edwardsiella ictaluri. Journal World Aquaculture Society, v.34, n.1, p.18-28, 2003.
MELO, J.F.B. et al. Desenvolvimento e composição corporal de alevinos de jundiá (Rhamdia quelen). Ciência Rural, Santa Maria, v.32, n.3, p.323-327, 2002.

PESTI, G.M.; MILLER, B.R. Animal feed formulation User Friendly Feed Formulation Program. Athens: Kluwer Academic Publishers Group, 1992. 166p.

RANZANI-PAIVA, M.J.T. et al. Hematological analysis in “cachara”, Pseudoplatystoma fasciatum Linneus, 1766, in captivity. In: WORLD AQUACULTURE, 2000, Nice, França. Anais... Oostende, Belgium: European Aquaculture Society, 2000a. V.28. p.590.

RANZANI-PAIVA, M.J.T. et al. Hematological evaluation in commercial fish species from the floodplain on the upper Paraná River, Brazil. Acta Scientiarum, v.22, n.2, p.515521, 2000b.

SILFVERGRIP, A.M.C. A systematic revision of the neotropical catfish genus Rhamdia. 1996. 156f. Thesis (PhD in Zoology) - Course of Zoology, Stockholm University.

STATISTICA for Windows [Computer program manual]. StatSoft, Version 1998, e-mail: info@statsoft.com. WEB:http://www.statsoft.com. On line. 2001.

TAVARES-DIAS, M. et al. Características hematológicas de teleósteos brasileiros. IV. Variáveis do jundiá (Rhamdia quelen) (Pimelodidae). Ciência Rural, v.32, p.693-698, 2002.

TAVARES-DIAS, M.; MORAES, F.R. Hematologia de peixes teleósteos. Ribeirão Preto: Villimpress, 2004. 144p.

WISE, D.J. et al. Effects of dietary selenium and vitamin E on red blood cell peroxidation, glutathione peroxidase activity, and macrophage superoxide anion production in channel catfish. Journal of Aquatic Animal Health, v.5, p.177182, 1993. 